\title{
Activating RNAs associate with Mediator to enhance chromatin architecture and transcription
}

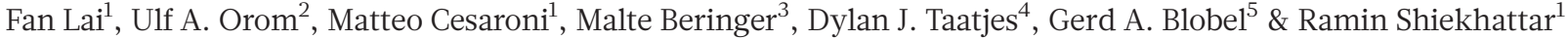

Recent advances in genomic research have revealed the existence of a large number of transcripts devoid of protein-coding potential in multiple organisms ${ }^{1-8}$. Although the functional role for long noncoding RNAs (lncRNAs) has been best defined in epigenetic phenomena such as $\mathrm{X}$-chromosome inactivation and imprinting, different classes of lncRNAs may have varied biological functions ${ }^{8-13}$. We and others have identified a class of lncRNAs, termed ncRNA-activating (ncRNA-a), that function to activate their neighbouring genes using a cis-mediated mechanism ${ }^{5,14-16}$. To define the precise mode by which such enhancer-like RNAs function, we depleted factors with known roles in transcriptional activation and assessed their role in RNA-dependent activation. Here we report that depletion of the components of the co-activator complex, Mediator, specifically and potently diminished the ncRNA-induced activation of transcription in a heterologous reporter assay using human HEK293 cells. In vivo, Mediator is recruited to ncRNA-a target genes and regulates their expression. We show that ncRNA-a interact with Mediator to regulate its chromatin localization and kinase activity towards histone $\mathrm{H} 3$ serine 10. The Mediator complex harbouring disease-causing MED12 mutations ${ }^{17,18}$ displays diminished ability to associate with activating ncRNAs. Chromosome conformation capture confirmed the presence of DNA looping between the ncRNA-a loci and its targets. Importantly, depletion of Mediator subunits or ncRNA-a reduced the chromatin looping between the two loci. Our results identify the human Mediator complex as the transducer of activating ncRNAs and highlight the importance of Mediator and activating ncRNA association in human disease.

To define the transcriptional complex(es) that orchestrate the responsiveness of activating lncRNAs, we used HEK293 stable cell lines expressing the heterologous TK promoter driving luciferase expression fused to ncRNA-a7 (also called LINC00651) controlled by its own natural promoter and depleted factors known to be involved in transcriptional activation and enhancer function. We also developed stable lines expressing control reporters, in which a DNA fragment devoid of any transcriptional activity was substituted for the ncRNA-a7 genomic region (Fig. 1a). Importantly, depletion of ncRNA-a7 using two different short interfering RNAs (siRNAs) specifically decreased the transcription of the reporter construct containing ncRNA-a7 (Fig. 1b). Next, we depleted factors known to be involved in transcriptional activation (Supplementary Fig. 1a) and examined the responsiveness of cell lines harbouring the constructs expressing ncRNA-a7 or control constructs devoid of IncRNAs. Interestingly, only depletion of the Mediator subunit MED12 displayed a differential effect on the transcription of the reporter construct containing ncRNA-a7 (Fig. 1c). Depletion of other factors was either ineffective in changing transcriptional output (CDK9, CCNT1, NIPBL or WDR5) or reduced the transcriptional levels for both constructs (GTF2B, p300, SMC1) (Fig. 1c).

Mediator contains a complex and modular subunit composition ${ }^{19,20}$. We depleted different components of Mediator corresponding to the head, middle or the tail modules and assessed their effect on the responsiveness of the cell lines harbouring the constructs driven by ncRNA-a7 or the control construct. Overall, depletion of other subunits of the Mediator complex also reduced the ncRNA-a7-induced activation, although there was also a small reduction in transcription of the control plasmid with depletion of some of the subunits (Fig. 1d and
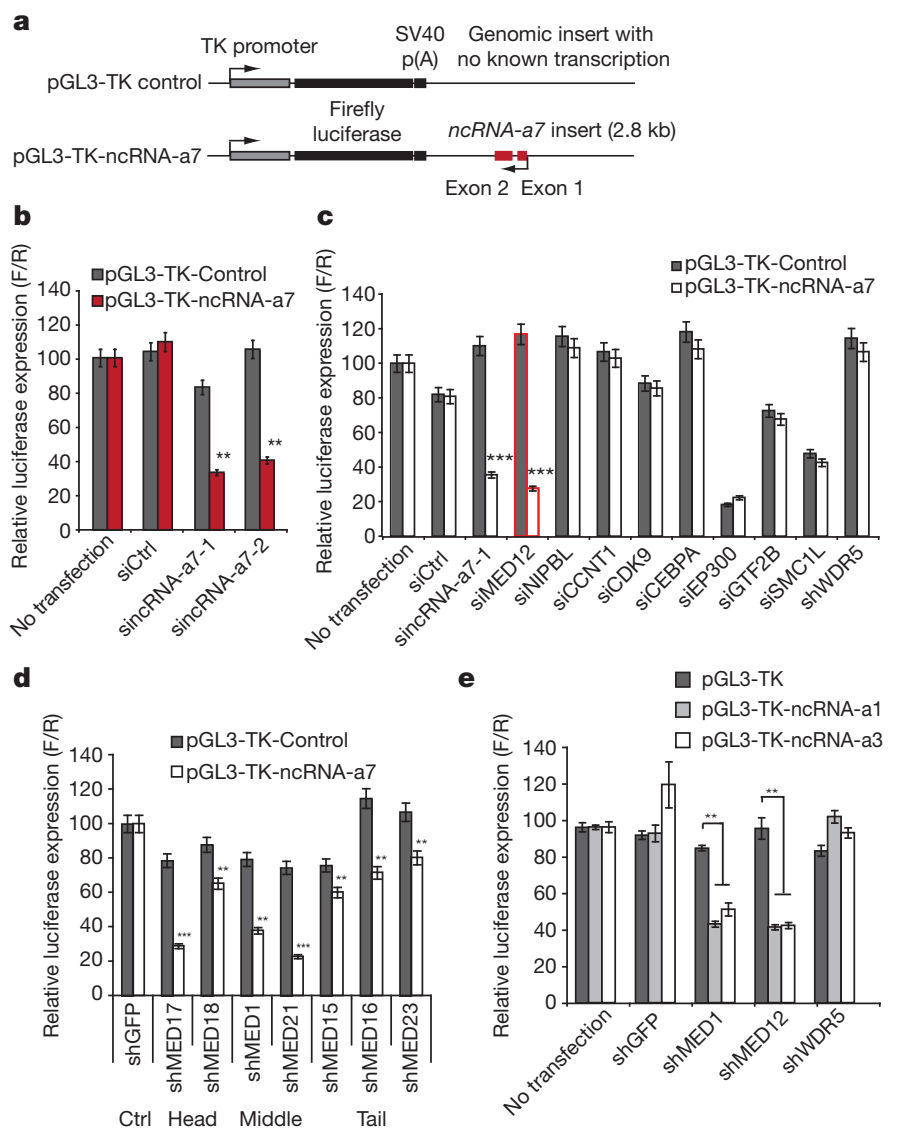

Figure 1 Mediator confers the ncRNA-a-dependent activation of a heterologous reporter. a, Schematic representation of genomic control insert (top) or $n c R N A-a 7$ insert (bottom) in luciferase reporter vector driven by a TK promoter. $\mathbf{b}$, Depletion of ncRNA-a7 reduces the luciferase activity in the ncRNA-a7 luciferase reporter cell lines. c, Depletion of the transcription factors or enhancers in the control or ncRNA-a7 reporter cell lines. The red bar indicates reduced transcription using siRNA against MED12, comparable with siRNA against $n c R N A-a 7$. d, Depletion of different Mediator subunits (head, middle and tail) using the ncRNA-a7 reporter cell lines. e, Depletion of the Mediator subunits in ncRNA-a1 or ncRNA-a3 luciferase reporter cell lines. All data shown are mean \pm s.e.m. of three independent experiments. $* * P<0.01$, $* * * P<0.001$ by two-tailed Student's $t$-test. $\mathrm{F} / \mathrm{R}$, firefly luciferase/Renilla luciferase.

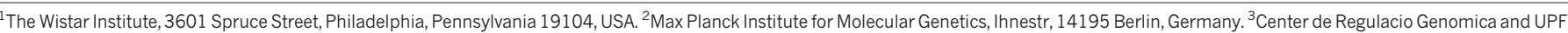

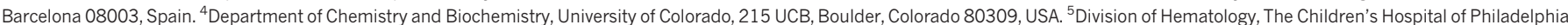
Philadelphia, Pennsylvania 19104, USA.
} 
Supplementary Fig. 1b). Similar to its effects on ncRNA-a7, depletion of the Mediator subunits decreased the RNA-induced activation of two other ncRNA-a, which were previously shown to regulate ECM1 and TAL1 genes (Fig. 1e). Taken together, these results identify the Mediator complex as a transducer of ncRNA-a function.

We next depleted the Mediator subunits and assessed their effect on ncRNA-a targets in vivo. We previously found that ncRNA-a7 and ncRNA-a3 regulate SNAI1 and TAL1 genes, respectively ${ }^{5}$. Moreover, using gene expression arrays, we had observed a profound decrease in AURKA transcription when ncRNA-a7 was depleted ${ }^{5}$. We confirmed these results by depleting ncRNA-a7 and ncRNA-a3, which led to a decrease in SNAI1, AURKA and TAL1 transcript levels using real-time PCR (Fig. 2a, b). We next depleted two different subunits of the Mediator complex, MED1 and MED12, and assessed the SNAI1, AURKA and TAL1 transcript levels (Supplementary Fig. 1b). Importantly, depletion of Mediator subunits decreased transcription of all three targets similar to that shown with depletion of ncRNA-a (Fig. 2a, b, the affected genes are depicted by a black bar). Interestingly, depletion of Mediator subunits did not affect the transcript levels of genes that are not regulated by ncRNA-a (UBE2V1, CSTF, STIL, depicted by a white bar in Fig. 2a, b). However, whereas ncRNA-a7 transcription was not affected after Mediator depletion, there was a decrease in ncRNA-a3 levels (Fig. 2a, b).

To ensure that such transcriptional effects after Mediator depletion were direct, we performed chromatin immunoprecipitation (ChIP) using antibodies against the MED12 and MED1 subunits of Mediator. This analysis revealed that the Mediator complex occupied the promoters of ncRNA-a3, ncRNA-a7 and their targets (Fig. 2c, d). Moreover, we found RNA polymerase II (RNAPII) at these promoters, consistent with Mediator occupancy (Fig. 2c, d). Importantly, depletion of ncRNA-a7 or ncRNA-a3 decreased the occupancy of Mediator and RNAPII at their target genes without affecting the occupancy at genes not regulated by ncRNA-a (Fig. 2c, d). These results indicate that the Mediator complex occupies the promoters of genes regulated by ncRNA-a, and depletion of ncRNA decreases the occupancy of Mediator at these sites.

The Mediator complex, via its CDK8 module, displays kinase activity towards histone $\mathrm{H} 3$ serine 10 (H3S10), a histone modification with strong ties to transcriptional activation ${ }^{21,22}$. We next assessed whether ncRNA-a regulate Mediator kinase activity towards histone H3. Addition of ncRNA-a7 or ncRNA-a3 led to a significant stimulation of the Mediator kinase activity towards histone $\mathrm{H} 3$, whereas the addition of primary let7b ncRNA or ncRNA HOTAIR had no effect (Fig. 2e, f and Supplementary Fig. 2a). Such stimulation of Mediator kinase activity by ncRNA-a was specific towards histone $\mathrm{H} 3$ as the substrate. We did not observe any stimulation when GST-CTD (corresponding to the $\mathrm{C}$-terminal domain of RPB1) or cyclin $\mathrm{H}$ was used as substrates (Supplementary Fig. 2b, c). Moreover, consistent with a role for ncRNA-a in stimulation of histone $\mathrm{H} 3$ phosphorylation, depletion of ncRNA-a7 or ncRNA-a3 led to a specific decrease in H3S10 levels at SNAI1, AURKA and TAL1 (Supplementary Fig. 2d and e).

We next asked whether ncRNA-a could associate with the Mediator complex. We isolated Mediator using stable cell lines expressing Flagepitope-tagged MED12 and examined its interaction with in-vitrotranscribed ncRNA-a7. An eluate from a stable Flag-GFP cell line was used as a control (Fig. 3a). Whereas the control primary let7 transcript (pri-let7) or the ncRNA HOTAIR (a 429-nucleotide RNA with a similar base composition as ncRNA-a7) did not specifically interact with the Mediator complex, we detected a robust and specific association between ncRNA-a7 and the Mediator complex (Fig. 3a). Notably, Mediator also associated with ncRNA-a 1 and ncRNA-a3, two other ncRNA-a that were shown to activate transcription of their neighbouring genes ${ }^{5}$ (Fig. 3b).

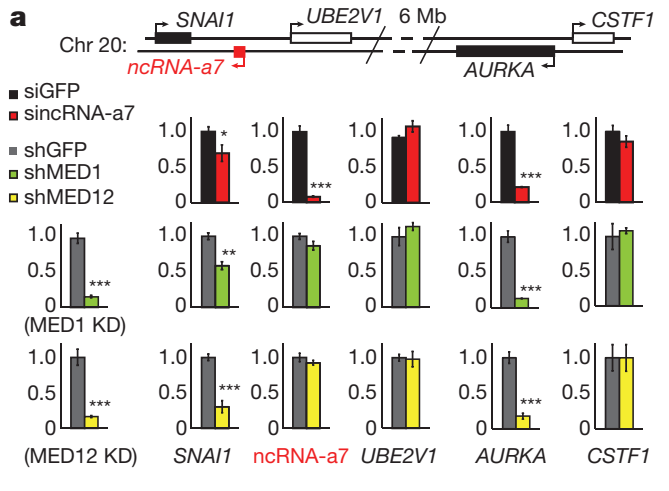

C $\square$ siCtrl $\square \operatorname{sincRNA-a7}$
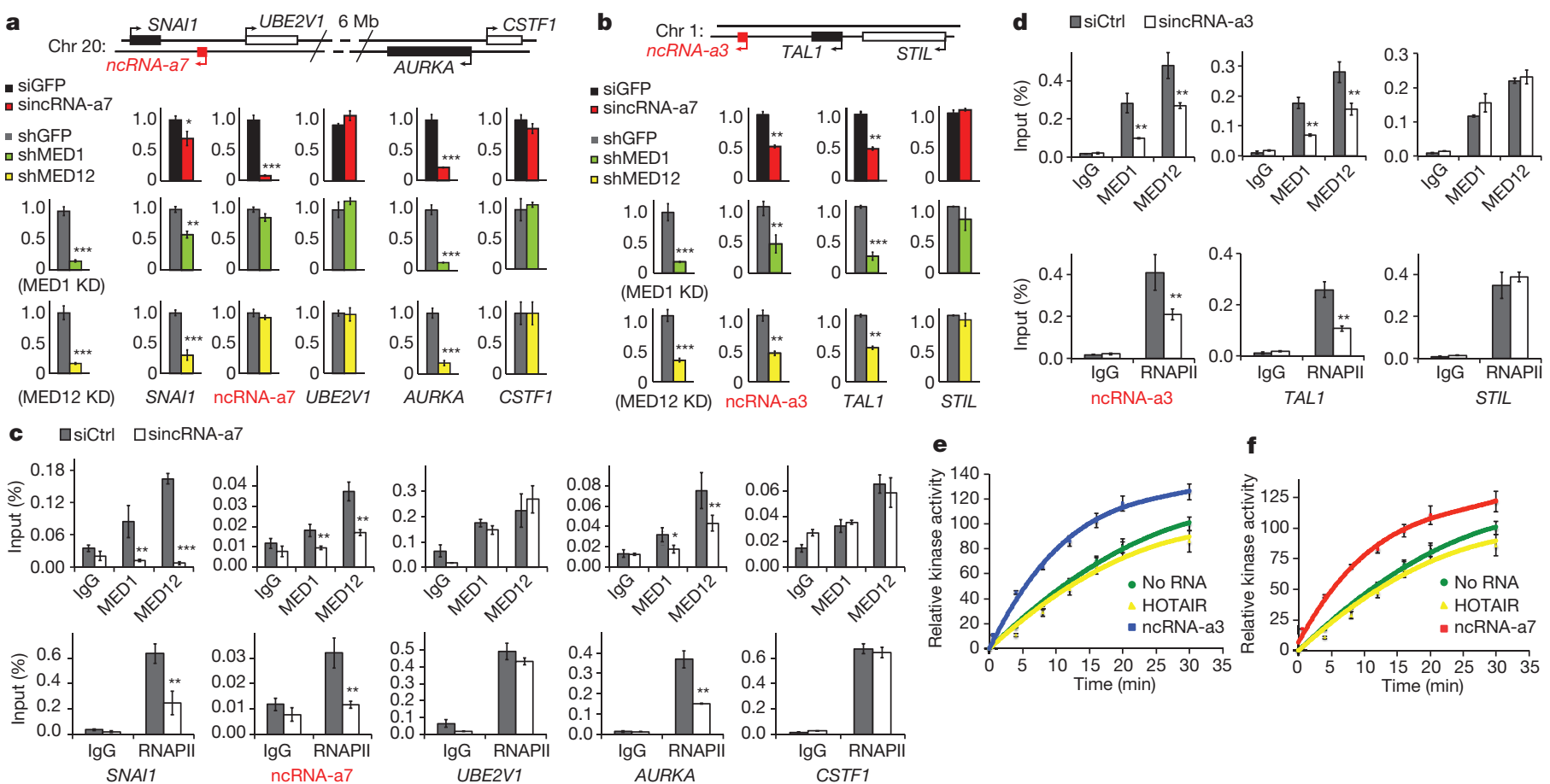

Figure 2 Functional association of Mediator and activating ncRNAs. a, Knockdown of ncRNA-a7 or Mediator subunits decreases expression of SNAI1 and AURKA. Depletion of ncRNA-a7 diminished SNAI1 and AURKA expression as detected by real-time PCR (top row, red bars). Similarly, knockdown (KD) of MED1 (green bars) or MED12 (yellow bars) reduced expression of SNAI1 or AURKA. b, Knockdown of ncRNA-a3 or Mediator subunits decreased TAL1 expression. Expression of TAL1 or control STIL after knockdown of ncRNA-a3 (red bars), MED1 (green bars) or MED12 (yellow bars) is shown. $\mathbf{c}, \mathbf{d}$, Knockdown of ncRNA-a7 (c) or ncRNA-a3 (d) reduces the

genomic occupancy of MED1, MED12 (top row) or RNAPII (bottom row) on SNAI1, AURKA (c) or TAL1 (d) in A549 cells. e, f, Activating ncRNAs specifically stimulate Mediator kinase activity towards histone H3.1 substrate in vitro. Quantification of kinase assay after addition of ncRNA-a7 (e) or ncRNA-a3 (f) is shown. Error bars represent \pm s.e.m. $(n=3), P<0.01$ by twotailed Student's $t$-test. The mean \pm s.e.m. for all results represents three independent experiments. $* P<0.05,{ }^{*} P<<0.01, * * * P<0.001$ by two-tailed Student's $t$-test. 
a

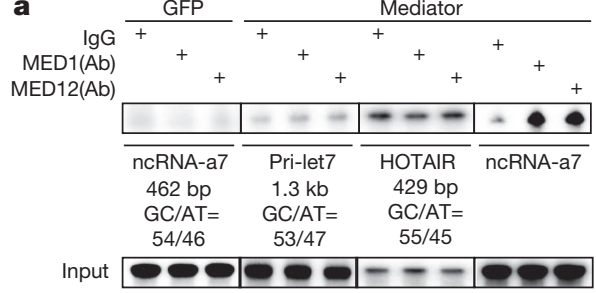

b
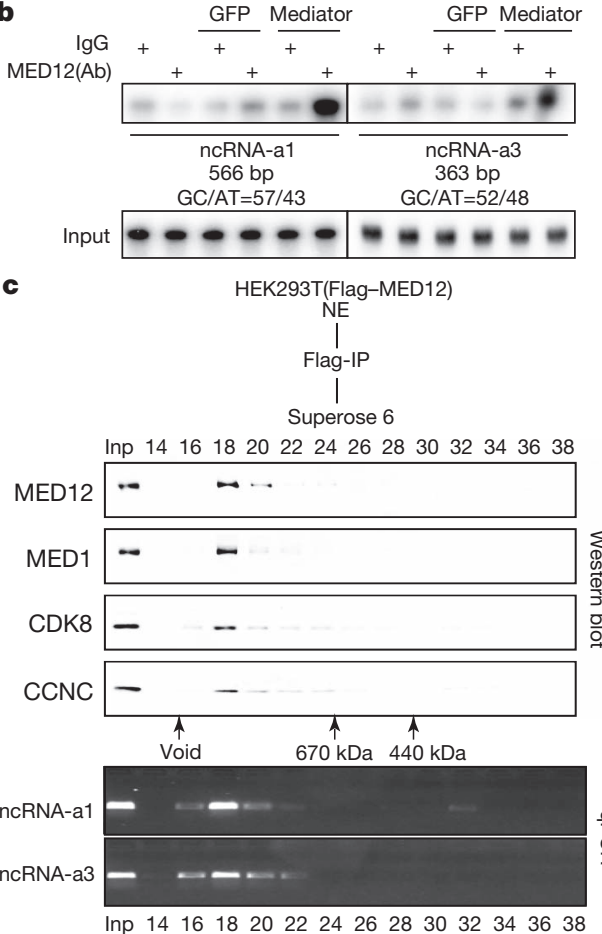

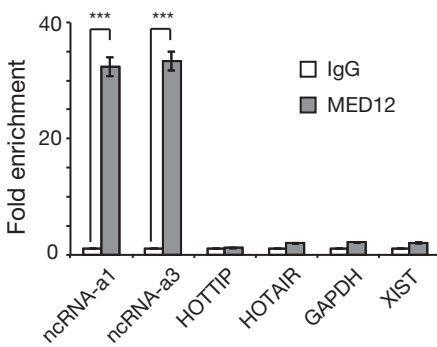

e

MED12 952 CGVVKHGMNRSD 963

MED12(G958E) 952 CGVVKHEMNRSD 963 MED12(R961W) 952 CGVVKHGMNWSD 693

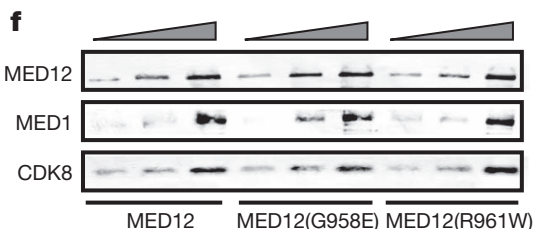

g

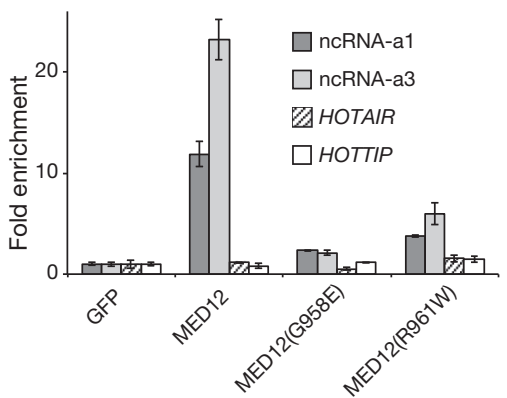

Figure 3 Interaction of Mediator and activating ncRNAs is disrupted by FG syndrome mutations of MED12. a, b, ncRNA-a associate with the Mediator complex in vitro. RNA immune precipitation (RIP) was performed using protein A dynabeads coupled with IgG, MED1 or MED12 antibodies. Identical results were obtained in three independent experiments. c, Purification of Mediator protein complex and associated RNAs. The purified Flag-MED12 affinity elution was subjected to Superose 6 size-exclusion chromatography and the Mediator subunits were detected by western blot. The Superose 6 fractions (14-38) are shown on top and molecular mass markers (kilodaltons) are at the bottom. The RNAs in each fraction were extracted followed by RT-PCR using specific primers. d, Ultraviolet crosslink followed by RIP using HEK293T whole-cell lysates. The associated RNAs were analysed by RT-qPCR with individual specific RNA primers. The mean \pm s.e.m. are from

To assess the association of endogenous ncRNA-a and Mediator, the Flag-MED12 affinity-purified Mediator was fractionated by gel filtration and column fractions were subjected to western blot analysis and polymerase chain reaction with reverse transcription (RT-PCR) to detect the associated ncRNA-a (Fig. 3c). Whereas the HEK293 nuclear extract contained similar levels of ncRNA-a1, ncRNA-a3, HOTAIR and HOTTIP (HEK293 cells do not express ncRNA-a7; Supplementary Fig. 3a), we could only detect ncRNA-a1 and ncRNA-a3 in association with the affinity-purified Mediator (Fig. 3c). Indeed, ncRNA-a1 and ncRNA-a3 displayed a co-elution with components of the Mediator complex on gel filtration, further supporting their association with Mediator (Fig. 3c). Next, we performed RNA immunoprecipitation following ultraviolet crosslinking (UV-RIP) with antiMED12 antibodies or IgG, as control. Activating ncRNA-a1 and ncRNA-a3 were significantly enriched in the MED12 UV-RIP, whereas other abundant control messenger RNAs or non-coding RNAs such as GAPDH, HOTAIR, HOTTIP and XIST were not (Fig. 3d). Two mutations in MED12 proteins have been linked to three independent experiments. ${ }^{* * *} P<0.001$ by two-tailed Student's $t$-test. e, Protein sequences of MED12 mutants causing FG syndrome. Part of the protein sequence of MED12 exon 21 is shown (top tow). The mutated sites indicate the G958E mutation (underlined) and the R961W mutation (underlined). f, HEK293T cells were transfected with Flag-MED12 or mutant constructs. After ultraviolet crosslinking, total cell extracts were immunoprecipitated and analysed by western blotting. g, After UV-RIP of wild-type and mutant MED12 samples shown in $\mathbf{f}, \mathrm{RT}-\mathrm{qPCR}$ was performed using transcript-specific primers from the lncRNAs shown. The data are representative of three independent experiments. The two-tailed Student's $t$-test indicated a $P<0.001$ for ncRNA-al and ncRNA-a3, whereas there was no significant difference for HOTAIR and HOTTIP.

the development of the human genetic disorder Opitz-Kaveggia syndrome (also know as FG syndrome), causing physical anomalies and developmental delays ${ }^{17,18}$ (Fig. 3e). Notably, whereas such mutations in MED12 do not affect its association with other Mediator subunits, they significantly diminish its association with ncRNA-a1 and ncRNA-a3 as measured by UV-RIP (Fig. 3f, g).

We next asked whether we could detect long-range chromatin looping between the ncRNA-a7 locus and its targets, SNAI1 and AURKA and whether ncRNA-a7 and the Mediator complex have a role in such an association. We performed chromosome conformation capture (3C) to assess the association of ncRNA-a7 with AURKA as well as SNAI1. We also performed a similar analysis between ncRNA-a3 and the TAL1 gene. We used anchoring points near the $3^{\prime}$ untranslated region of ncRNA-a7 or ncRNA-a3 to measure the extent of chromatin looping between the $n c R N A-a 7$ or $n c R N A-a 3$ locus and their targets as shown in Fig. 4a, d and Supplementary Fig. 3b, c. This analysis revealed a strong association between the ncRNA-a7 locus and the region encompassing the promoter region of SNAI1 (Fig. 4b, upper panel). 


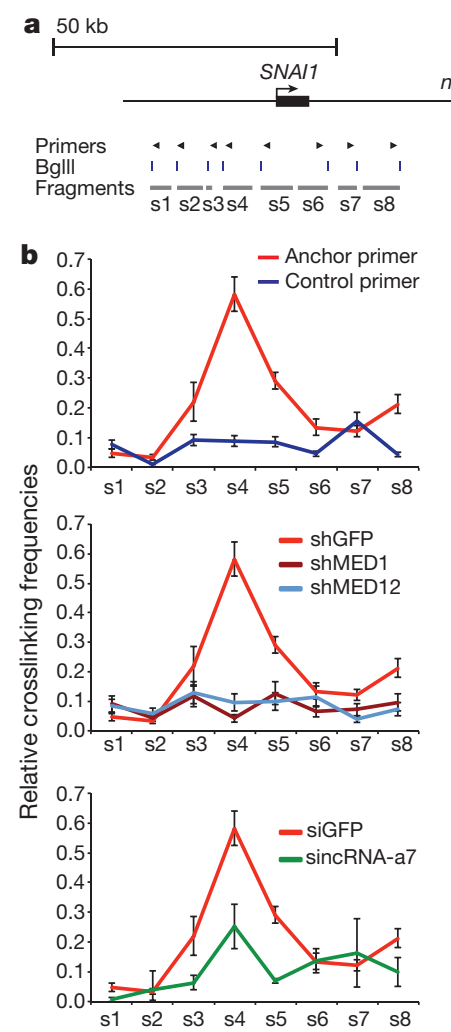

Figure $4 \mid$ Mediator complex and ncRNA-a promote chromatin looping. The schematic diagrams represent the genomic locus between the $n c R N A-\mathrm{a} 7$, SNAI1 and AURKA loci (a), and the ncRNA-a3 and TAL1 loci (d). The top arrows show the position of primers, the digestion sites are shown in the middle, and the s1-s8, A-G or 1-8 fragments are presented below. The looping events between ncRNAs and their targets were detected between ncRNA-a7 and SNAI1 (b), ncRNA-a7 and AURKA (c) and ncRNA-a3 and TAL1 (e) using chromosome conformation capture (3C). Depletion of MED1 or MED12

Moreover, ncRNA-a7 also displayed an interaction with regions near the $5^{\prime}$ end of the AURKA gene that extended into the body of the gene (Fig. 4c, upper panel). There was no detectable association when a control anchor was placed in the genomic region between $n c R N A-a 7$ and AURKA (Fig. 4b, c).

Next we depleted the Mediator subunits MED1 or MED12 and assessed their potential role in mediating the chromosomal looping between ncRNA-a7, SNAI1 and AURKA. Depletion of either MED1 or MED12 completely abrogated the chromosomal looping between ncRNA-a7 and SNAI1 (Fig. 4b, middle panel). A similar result was obtained after depletion of MED1 or MED12 at the AURKA locus (Fig. 4c, middle panel). Importantly, depletion of ncRNA-a7 had a comparable effect, reducing the chromosomal looping between ncRNA-a7 and both SNAI1 and AURKA loci (Fig. 4b, c, lower panels). Because depletion of ncRNA-a7 using siRNAs resulted in a partial decrease of ncRNA-a7 concentration (about 60\% depletion, Supplementary Fig. 1), the residual ncRNA-a7 may be sufficient to promote smaller levels of DNA looping seen after ncRNA-a7 depletion.

We also performed a similar analysis measuring the extent of DNA looping between ncRNA-a3 and that of the TAL1 locus. Similar to results obtained with ncRNA-a7, we detected a specific and robust DNA looping between ncRNA-a3 and TAL1 (Fig. 4e). Finally, depletion of Mediator subunits MED1 and MED12 or ncRNA-a3 nearly abolished the DNA looping between ncRNA-a3 and TAL1 (Fig. 4e, middle and lower panels). Notably, Mediator and ncRNA-a association with their target genes extend beyond the transcription start sites to include the more distal sites. This may reflect the engagement of proximal regulatory elements in ncRNA-a and Mediator chromatin looping (Fig. 4b, e). These results suggest a mechanism of action for

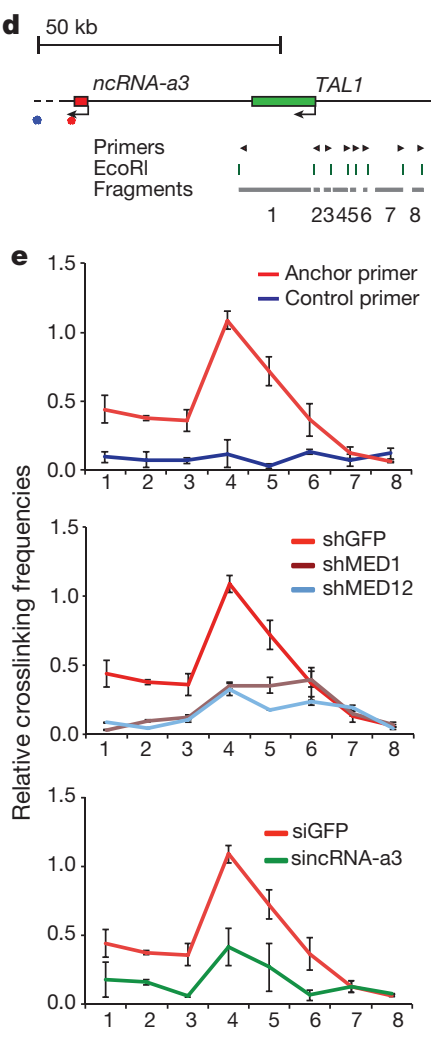

abolished the loop interaction (b, c, e; middle panels). Knockdown of ncRNAa7 or ncRNA-a3 reduced the chromosomal looping events (b, c, e; lower panels). The interaction frequency between the anchoring points and distal fragments was determined by real-time PCR and normalized to BAC templates and control anchors. Each error bar represents \pm s.e.m. from three independent experiments, $P<0.01$ by two-tailed Student's $t$-test.

Representative gel images of the $3 \mathrm{C}$ experiments for AURKA are presented in Supplementary Fig. 3.

ncRNA-a and Mediator that may involve additional chromatin contacts beyond that which has been observed for distal regulatory elements and transcriptional start sites.

We propose a new mechanism of action for a class of lncRNAs termed ncRNA-activating (ncRNA-a) involved in long-range transcriptional activation through their association with the Mediator complex (Supplementary Fig. 4). It is noteworthy that such MediatorncRNA-a-dependent chromatin loops extend beyond the transcription start sites of the target promoters, which may reflect the inclusion of promoter proximal regulatory elements in such associations. Future studies could also assess whether such ncRNA-a-Mediator chromatin loops augment, or function exclusively of, Mediator-Cohesin complexes $^{23}$.

Recently, experiments in zebrafish uncovered a fundamental role for IncRNAs in developmental control ${ }^{24}$. Moreover, recent reports have linked a number of lncRNAs to human diseases such as cancer and neurological disorders ${ }^{11,25-27}$. Importantly, our studies indicated that Mediator complexes containing disease-causing mutant MED12 proteins corresponding to FG syndrome fail to associate with ncRNAa. These results underscore the significance of Mediator-ncRNA-a association and suggest the loss of such Mediator-ncRNA-a interactions as a possible contributing factor in such developmental disorders.

\section{METHODS SUMMARY}

Depletion experiments using siRNA/DNA transfection and ncRNA luciferase assays. pGL3-TK-ncRNA-a and pGL3-TK-control constructs were generated as described previously. On-TargetPlus siRNAs and non-targeting control siRNA were purchased from Dharmacon. The modified siDNA oligonucleotides were purchased from Integrated DNA Technologies. siRNA/DNA transfections were 
performed with Lipofectamine RNAiMAX (Life Technologies) according to the manufacturer's instructions, using $50 \mathrm{nM}$ siRNA/DNAs in a final volume of $2 \mathrm{ml}$ of culture medium without antibiotics. Cells were collected $48-72 \mathrm{~h}$ after two rounds of transfection.

Chromatin immunoprecipitation analysis was performed using $1 \times 10^{7}$ to $3 \times 10^{7}$ A549 or MCF7 cells were collected for each independent immunoprecipitation. After chemically crosslinking, cells were fixed in $1 \%$ formaldehyde $1 \times$ PBS with $10 \%$ serum for 15 min at room temperature. After immunoprecipitation, bound chromatin was eluted at $65^{\circ} \mathrm{C}$ for $10 \mathrm{~min}$ in $60 \mu \mathrm{l}$ fresh elution buffer twice (TE, $1 \%$ SDS) and $80 \mu \mathrm{l}$ of elution buffer was added to the final volume of 200 $\mu$ l. Crosslinking samples were reversed overnight at $65^{\circ} \mathrm{C}$. After proteinase $\mathrm{K}$ treatment $\left(0.5 \mathrm{mg} \mathrm{ml}^{-1}\right)$, DNA was purified using a QIAquick PCR purification kit (Qiagen).

Protein affinity purification and RNA immunoprecipitation. The affinitypurified mediator complex and the GFP protein control were generated from the Flag-tagged MED12 or GFP HEK293T stable cell lines. RNA probes, internally labelled with $\left[\alpha_{-}{ }^{32} \mathrm{P}\right] \mathrm{CTP}$, were transcribed using T7 or Sp6 RNA polymerase (Riboprobe System, Promega). RNA immunoprecipitations were performed using Dynabeads Protein A (Invitrogen) according to the manufacturer's recommendations. Transient transfected HEK293T cells were UV-crosslinked at $254 \mathrm{~nm}$ $\left(200 \mathrm{~mJ} \mathrm{~cm}^{-2}\right)$ in $10 \mathrm{ml}$ ice-cold PBS and collected by scraping. Cells were incubated in lysis solution $(0.1 \%$ SDS, $0.5 \%$ NP40, 0.5\% sodium deoxycholate, $400 \mathrm{U} \mathrm{ml}^{-1}$ RNase inhibitor (Roche)), and protease inhibitor at $4{ }^{\circ} \mathrm{C}$ for $25 \mathrm{~min}$ with rotation, followed by DNase treatment $\left(30 \mathrm{U}\right.$ of DNase, 15 min at $\left.37^{\circ} \mathrm{C}\right)$.

Full Methods and any associated references are available in the online version of the paper.

\section{Received 29 March; accepted 31 December 2012.}

Published online 17 February 2013.

1. Birney, E. et al. Identification and analysis of functional elements in $1 \%$ of the human genome by the ENCODE pilot project. Nature 447, 799-816 (2007).

2. Bertone, P. et al. Global identification of human transcribed sequences with genome tiling arrays. Science 306, 2242-2246 (2004)

3. The FANTOM Consortium. The transcriptional landscape of the mammalian genome. Science 309, 1559-1563 (2005).

4. Okazaki, Y. et al. Analysis of the mouse transcriptome based on functional annotation of 60,770 full-length cDNAs. Nature 420, 563-573 (2002).

5. Ørom, U.A. etal. Long noncoding RNAs with enhancer-like function in human cells Cell 143, 46-58 (2010)

6. Guttman, M. et al. Chromatin signature reveals over a thousand highly conserved large non-coding RNAs in mammals. Nature 458, 223-227 (2009).

7. Kapranov, P. et al. RNA maps reveal new RNA classes and a possible function for pervasive transcription. Science 316, 1484-1488 (2007).

8. Wang, K. C. \& Chang, H. Y. Molecular mechanisms of long noncoding RNAs. Mol. Cell 43, 904-914 (2011)

9. Lee, J. T. The $X$ as model for RNA's niche in epigenomic regulation. Cold Spring Harb. Perspect. Biol. 2, a003749 (2010).

10. Rinn, J. L. et al. Functional demarcation of active and silent chromatin domains in human HOX loci by noncoding RNAs. Cell 129, 1311-1323 (2007)

11. Gupta, R. A. et al. Long non-coding RNA HOTAIR reprograms chromatin state to promote cancer metastasis. Nature 464, 1071-1076 (2010).
12. Yap, K. L. et al. Molecular interplay of the noncoding RNA ANRIL and methylated histone $\mathrm{H} 3$ lysine 27 by polycomb CBX7 in transcriptional silencing of INK4a. Mol. Cell 38, 662-674 (2010).

13. Zhao, J. et al. Genome-wide identification of polycomb-associated RNAs by RIPseq. Mol. Cell 40, 939-953 (2010).

14. Kim, T. K. et al. Widespread transcription at neuronal activity-regulated enhancers. Nature 465, 182-187 (2010).

15. De Santa, F. et al. A large fraction of extragenic RNA pol II transcription sites overlap enhancers. PLoS Biol. 8, e1000384 (2010)

16. Wang, K. C. et al. A long noncoding RNA maintains active chromatin to coordinate homeotic gene expression. Nature 472, 120-124 (2011).

17. Risheg, H. et al. A recurrent mutation in MED12 leading to R961W causes OpitzKaveggia syndrome. Nature Genet. 39, 451-453 (2007).

18. Rump, P. et al. A novel mutation in MED12 causes FG syndrome (Opitz-Kaveggia syndrome). Clin. Genet. 79, 183-188 (2011)

19. Malik, S. \& Roeder, R. G. The metazoan Mediator co-activator complex as an integrative hub for transcriptional regulation. Nature Rev. Genet. 11, 761-772 (2010)

20. Taatjes, D. J. The human Mediator complex: a versatile, genome-wide regulator of transcription. Trends Biochem. Sci. 35, 315-322 (2010).

21. Knuesel, M. T., Meyer, K. D., Donner, A. J., Espinosa, J. M. \& Taatjes, D. J. The human CDK8 subcomplex is a histone kinase that requires Med12 for activity and can function independently of mediator. Mol. Cell. Biol. 29, 650-661 (2009).

22. Nowak, S. J.\& Corces, V. G. Phosphorylation of histone H3: a balancing act between chromosome condensation and transcriptional activation. Trends Genet. 20, 214-220 (2004)

23. Kagey, M. H. et al. Mediator and cohesin connect gene expression and chromatin architecture. Nature 467, 430-435 (2010)

24. Ulitsky, I, Shkumatava, A. Jan, C. H. Sive, H. \& Bartel, D. P. Conserved function of lincRNAs in vertebrate embryonic development despite rapid sequence evolution. Cell 147, 1537-1550 (2011).

25. Yu, W. et al. Epigenetic silencing of tumour suppressor gene $\mathrm{p} 15$ by its antisense RNA. Nature 451, 202-206 (2008).

26. Sopher, B. L. et al. CTCF regulates ataxin-7 expression through promotion of a convergently transcribed, antisense noncoding RNA. Neuron 70, 1071-1084 (2011).

27. Cabianca, D. S. et al. A long ncRNA links copy number variation to a polycomb/ trithorax epigenetic switch in FSHD muscular dystrophy. Cell 149, 819-831 (2012).

Supplementary Information is available in the online version of the paper.

Acknowledgements We thank Shiekhattar laboratory members for support and discussions. We thank W. Dang (Blobel laboratory) and I. Tempera for suggestions and technical help on $3 \mathrm{C}$ experiments. R.S. was partially supported by a cancer core grant from NIH (P30 CA 010815). D.J.T. was supported by a grant from the NCI (R01 CA127364). G.A.B. was supported by 5R37DK058044.

Author Contributions R.S., F.L. and U.A.O. conceived and designed the overall project with help from G.A.B., M.B., D.J.T. and M.C. F.L. and U.A.O. performed the RNAi screens; F.L. received advice from G.A.B. to execute the 3 C experiments. F.L. and M.C. performed the ChIP experiments for Mediator. F.L. performed all experiments regarding the ncRNA association with Mediator and the kinase assays. F.L., M.C. and R.S. analysed the data and wrote the paper.

Author Information Reprints and permissions information is available at www nature.com/reprints. The authors declare no competing financial interests. Readers are welcome to comment on the online version of the paper. Correspondence and requests for materials should be addressed to R.S. (shiekhattar@wistar.org). 


\section{METHODS}

siRNA/DNA transfection and ncRNA luciferase assays. pGL3-TK-ncRNA-a and pGL3-TK-control constructs were generated as described previously. Firefly luciferase constructs, pRL-CMV and a selectable marker for puromycin resistance were co-transfected in HEK293T cells. Transfected cells were grown in the presence of $2.5 \mu \mathrm{g} \mathrm{ml}^{-1}$ puromycin (Sigma) for selection. Individual colonies were isolated and screened for luciferase expression. Luciferase assays were performed in 96-well white plates using Dual-Glo luciferase assay system (Promega) according to the manufacturer's protocol.

On-TargetPlus siRNAs and non-targeting control siRNA were purchased from Dharmacon. The modified siDNA oligonucleotides were purchased from Integrated DNA Technologies. siRNA or siDNA transfections were performed with Lipofectamine RNAiMAX (Life Technologies) according to the manufacturer's instructions, using $50 \mathrm{nM}$ siRNA/DNAs in a final volume of $2 \mathrm{ml}$ of culture medium without antibiotics. Cells were collected $48-72 \mathrm{~h}$ after two rounds of transfection.

Corresponding ncRNA-a HEK293T cell lines growing in 12-well dishes were transfected with corresponding siRNA oligonucleotides, using Metafectene Pro (Biontex Laboratories). Twenty-four hours after transfection, the cells were lysed in $200 \mu \mathrm{l}$ passive lysis buffer (Promega), and firefly and Renilla luciferase activities were measured on $30 \mu \mathrm{l}$ lysate using the Dual-Luciferase reporter assay kit (Promega). Individual experiments were realized in triplicate, and the ratios presented are the averages of three independent experiments.

siRNA sequences. siControl1: ON-TARGET non-targeting siRNA 1 (D-001810 01); siControl2: ON-TARGET Non-targeting siRNA 2 (D-001810-02); siGFP. GFP Duplex I (P-002048-01); sincRNA-a3-1: 5' -CUAUGGAAUUUAAGCCCA AUU-3'; sincRNA-a3-2: 5' -GCAAGAUCACGGUGCGUCAUU-3'; sincRNAa7-1: 5' -CCGAUUUGAGAGAGUGAGAUU-3'; sincRNA-a7-2: 5' -GAAGGGA ACCAGUGCUAAAUU- ${ }^{\prime}$; siMED12: 5' -UCACUCAUCUCAUGUUAUAUU 3'; siNIPBL: 5' -GGGAAUAUGAAGAGCGUGAUU-3' ; siCCNT1: 5' -GAACAA ACGUCCUGGUGAUUU-3'; siCDK9: $5^{\prime}$-UGACGUCCAUGUUCGAGUAUU3'; siCEBPA: 5' -ACAAGAACAGCAACGAGUAUU-3'; siEP300: 5' -GGACUA CCCUAUCAAGUAAUU-3'; siGTF2B: 5' -ACAAUCAGACAGUCCUAUAUU3'; siSMC1L: 5' -CAUCAAAGCUCGUAACUUCUU-3'

siDNA sequences. siGFP: $5^{\prime}-\mathrm{T}^{*} \mathrm{C}^{*} \mathrm{~A}^{*} \mathrm{C}^{*} \mathrm{C}^{*} \mathrm{~T}^{*} \mathrm{~T}^{*} \mathrm{C}^{*} \mathrm{~A}^{*} \mathrm{C}^{*} \mathrm{C}^{*} \mathrm{C}^{*} \mathrm{~T}^{*} \mathrm{C}^{*} \mathrm{~T}^{*} \mathrm{C}^{*} \mathrm{C}^{*}$ $\mathrm{A}^{*} \mathrm{C}^{*} \mathrm{~T}-3^{\prime}$; sincRNA-a1: $5^{\prime}-\mathrm{C}^{*} \mathrm{C}^{*} \mathrm{~T}^{*} \mathrm{G}^{*} \mathrm{C}^{*} \mathrm{C}^{*} \mathrm{C}^{*} \mathrm{~T}^{*} \mathrm{C}^{*} \mathrm{~T}^{*} \mathrm{~T}^{*} \mathrm{C}^{*} \mathrm{C}^{*} \mathrm{~T}^{*} \mathrm{C}^{*} \mathrm{C}^{*} \mathrm{~T}^{*} \mathrm{G}^{*}$ T*A-3'; sincRNA-a3: $5^{\prime}-G^{*} C^{*} T^{*} C^{*} G^{*} T^{*} C^{*} G^{*} G^{*} G^{*} T^{*} A^{*} C^{*} T^{*} C^{*} T^{*} C^{*} C^{*} A^{*}$

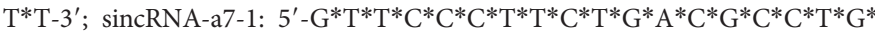
G*T*A-3'; sincRNA-a7-2: $5^{\prime}-\mathrm{C}^{*} \mathrm{~A}^{*} \mathrm{~T}^{*} \mathrm{C}^{*} \mathrm{~T}^{*} \mathrm{~T}^{*} \mathrm{C}^{*} \mathrm{~T}^{*} \mathrm{C}^{*} \mathrm{C}^{*} \mathrm{G}^{*} \mathrm{~T}^{*} \mathrm{C}^{*} \mathrm{~T}^{*} \mathrm{C}^{*} \mathrm{~T}^{*} \mathrm{G}^{*}$ $\mathrm{C}^{*} \mathrm{C}^{*} \mathrm{~A}^{*} \mathrm{C}-3^{\prime}$. Asterisks indicate nucleoside phosphorothioates.

shRNA infections. pLKO.1 lentiviral vector containing short hairpin RNAs (shRNAs) or shRNA targeting GFP control was obtained from Open Biosystems. The TRC shRNA constructs were purchased from Open Biosystems using Lentiviral packaging system. Twenty-four hours after infection, selection with $2.5 \mu \mathrm{g} \mathrm{ml}^{-1}$ puromycin was performed for $48 \mathrm{~h}$.

shRNA list. shGFP: RHS4459; MED12 (TRCN0000018576): 5'-CGGGT ACTTCATACTTTGG-3'; MED17 (TRCN0000019245): 5'-CCGAGCTTGC AGTTATCTATT-3'; MED18 (TRCN0000053164): 5'-GCCAGAAATGGG AGACAAGAA-3'; MED1 (TRCN0000019800): 5'-GCCGAGTTCCTCTTA TCCTAA-3'; MED21 (TRCN0000013429): 5'-CCATTGGAGTATTGCA GCAAT-3'; MED15 (TRCN0000018969): 5'-GCTCAGAACCAACCATCAC3'; MED16 (TRCN0000022139): 5'-CGACATTGACAAGGTCATGAT-3'; MED23 (TRCN0000019196): 5'-CGCAGTTTACACGCTTCCT-3'; WDR5 (TRCN0000118050): 5' -CCAACCTTATTGTCTCAGGAT-3'.

Antibodies and recombinant proteins. Antibodies and recombinant proteins used were: Pol II (SC-899, Santa Cruz), MED1 (A300-793A, Bethyl labs), MED12 (A300-774A, Bethyl labs), histone H3Ser10p (05-598, Millipore), CDK8 (SC-1521, Santa Cruz), CCNC (cyclin C, SC-1061, Santa Cruz), human rHistone H3.1 (M2503S, NEB), and human rCCNH (pka-364, Prospec protein specialists).

RNA purification, cDNA synthesis and quantitative PCR. Cells were collected and re-suspended in TRIzol (Invitrogen) and RNA extracted according to the manufacturer's protocol. cDNA synthesis was done using random primers with Fermentas RevertAid first-strand cDNA synthesis kit. Quantitative PCR was done using IQ SYBR green super-mixes and CFX96 Touch Real-Time PCR detection system (Bio-rad). For all quantitative PCR reactions GAPDH was measured for an internal control and used to normalize the data. $\mathrm{qPCR}$ primer sequences are listed here: ncRNA-a1-qfor: 5'-GCAAGCGGAGACTTGTCTTT-3'; ncRNA-a1-qrev: 5'-GGCTGGTCTTGAACTCCTGA-3'; ncRNA-a3-qfor: 5' -TTAAGCCCAAG GAATGGAGA-3'; ncRNA-a3-qrev: 5'-AGCGGTGTGGAATAAACTGG-3'; ncRNA-a7-q1for: 5' -ATCCTGGTGGAAAAGGCATC-3'; ncRNA-a7-q1 rev: 5' GCCTGGGAAAAGCTACTTCA-3'; ncRNA-a7-q2for: 5'-CCGTTGGCTCCA CAAACCT-3'; ncRNA-a7-q2rev: 5'-CAGTGACAGTAGCAGGCATCCT-3'; MED17-qfor: 5'-CCCAGCGGATAGACTTCAGC-3'; MED17-qrev: 5' -ATTGT
TCCTCACTGAGTCCCA-3'; MED18-qfor: 5'-GGGCACCATTAACATGAT GGA-3'; MED18-qrev: 5'-TGGTCAAGGAAAGTCTCAGGT-3'; MED1-qfor: 5'-CTGGAACGGCTCCATGCAA-3'; MED1-qrev: 5'-CTTCTCCATGACTTG ACGCAC-3'; MED21-qfor: 5' -TTGCACGAACAGCAAAAGACA-3'; MED21qrev: 5'-TGCGCTTTGTATCTTCTCCAG-3'; MED15-qfor: 5'-ATGGACG TTTCCGGGCAAG-3'; MED15-qrev: 5'-GCATCCTCGATTTGACTGACCA3'; MED16-qfor: 5' -TGCTGGACATGAACACACTG-3'; MED16-qrev: 5' -AGG GAACCCTGGTTGGGTA-3'; MED23-qfor: 5'-GGGCCTTCAGACAGTTTTG G-3'; MED23-qrev: 5'-AGCTGTGTTCTTTCCCACTCA-3'; MED12-qfor: 5' CTCCCGATGTTTACCCTCAG-3'; MED12-qrev: 5'-ATGCTCATCCCCAGA GACAG-3'; NIPBL-qfor: 5'-CGGCCCGGATTATAGTCTCT-3'; NIPBL-qrev: 5'-TCAGGTGCCAGCTGTCATTA-3'; CCNT1-qfor: 5'-GCCAATCTGCTTC AGGACA-3'; CCNT1-qrev: 5'-GGGAACTGTGTGAAGGACTGA-3'; CDK9qfor: 5'-ATACGAGAAGCTCGCCAAGA-3'; CDK9-qrev: 5'-CCTTCTCGT TTTCCATCAGC-3'; CEBPA-qfor: 5'-CAGAGGGACCGGAGTTATGA-3'; CEBPA-qrev: 5' -TTCACATTGCACAAGGCACT-3'; EP300-qfor: 5' -GGGAGG ACAAACAGGATTGA-3'; EP300-qrev: 5' -CCAATCTGCTGTCCAGGATT-3'; GTF2B-qfor: 5' -TTCCTGCTTTCGGTGTGTCT-3'; GTF2B-qrev: 5' -TGGATG GTTTGGACATGTGA-3'; SMC1L1-qfor: 5' -TCGGACCATTTCAGAGGTTC3'; SMC1L1-qrev: 5'-GGTCTTTACCCGCAGGTTG-3'; WDR5-qfor: 5'-AAT TCAGCCCGAATGGAGAGT-3'; WDR5-qrev: 5'-GGATATTCCCAGCTTGT GACC-3'; CSTF1-qfor: 5' -ACAGAACCAAAGTGGGCTTGA-3'; CSTF1-qrev: 5' -ACACAGACTGAGGCTTGATTTC-3'; AURKA-qfor: 5' -TTCAGGACCTG TTAAGGCTACA-3'; AURKA-qrev: 5'-ATTTGAAGGACACAAGACCCG-3'; TAL1-qfor: 5'-CAGCCTAGTGGCTTGTCCTC-3'; TAL1-qrev: 5'-GGAGC CTGAAATTGAATGGA-3'; HOTAIR-qfor: 5' -GGTAGAAAAAGCAACC ACGAAGC-3'; HOTAIR-qrev: 5' -ACATAAACCTCTGTCTGTGAGTGCC-3' HOTTIP-qfor: 5' -CCTAAAGCCACGCTTCTTTG-3'; HOTTIP-qrev: TGCAG GCTGGAGATCCTACT-3'; STIL-qfor: 5' -CCCAACGCCAACTGGAGATTT3'; STIL-qrev: 5'-AGTCGGATGGTCTTCTCAGTC-3'; SNAI1-qfor: 5' -TCGG AAGCCTAACTACAGCGA-3'; SNAI1-qrev: 5' -AGATGAGCATTGGCAGCG AG-3'; UBE2V1-qfor: 5'-AAGCAAGAGCGACGCAAGAT-3'; UBE2V1-qrev: 5'-CGGAAATTGCGAGGGACTT-3'.

Chromatin immunoprecipitation. Briefly, $1 \times 10^{7}$ to $3 \times 10^{7}$ A549 or MCF7 cells were collected for each independent immunoprecipitation. After chemically crosslinking, cells were fixed in $1 \%$ formaldehyde, $1 \times$ PBS with $10 \%$ serum for $15 \mathrm{~min}$ at room temperature. After quenching with $0.25 \mathrm{M}$ glycine, rinsing twice with cold $1 \times$ PBS, cells were lysed for $15 \mathrm{~min}$ in cold Chip buffer $(150 \mathrm{mM} \mathrm{NaCl}$, 1\% Triton X-100, 5 mM EDTA; 10 mM Tris-HCl, pH 7.5, 0.5 mM DTT) with protease inhibitors on ice for $10 \mathrm{~min}$. DNA was sonicated to between 200 - and 350-bp DNA fragments on a Diagenode Bioruptor according to manufacturer's protocol. The solubilized chromatin was cleared and diluted with lysis buffer (without SDS to bring down SDS concentration to $0.1-0.15 \%$ ). $1 \mathrm{ml}$ of the chro matin solution was incubated overnight with $2-6 \mu \mathrm{g}$ of antibody using protein A Dyna Beads at $4{ }^{\circ} \mathrm{C}$. The beads were washed twice with Mixed Micelle wash buffer (0.2\% SDS, $1 \%$ Triton X-100, 5.2\% w/v sucrose, 5 mM EDTA, $20 \mathrm{mM}$ Tris-HCl, $\mathrm{pH} 8,150 \mathrm{mM} \mathrm{NaCl}$ ), 2 times with high-salt buffer (1\% Triton X-100, $1 \mathrm{mM}$ EDTA, $50 \mathrm{mM}$ HEPES, pH 8, 0.1\% w/v deoxycholate, $500 \mathrm{mM} \mathrm{NaCl}$ ), 2 times with $\mathrm{LiCl}$ buffer $(250 \mathrm{mM} \mathrm{LiCl}, 0.5 \% \mathrm{NP}-40,0.5 \%$ deoxycholate, $1 \mathrm{mM}$ EDTA $10 \mathrm{mM}$ Tris- $\mathrm{HCl}, \mathrm{pH} 8)$, and one time with cold TE buffer $(10 \mathrm{mM}$ Tris- $\mathrm{HCl}$, $\mathrm{pH} 7.6,1 \mathrm{mM}$ EDTA). Bound chromatin was eluted at $65^{\circ} \mathrm{C}$ for $10 \mathrm{~min}$ in $60 \mu \mathrm{l}$ fresh elution buffer twice (TE, $1 \%$ SDS) and brought to $200 \mu$ l. Crosslinking samples were reversed overnight at $65^{\circ} \mathrm{C}$. After proteinase $\mathrm{K}$ treatment $\left(0.5 \mathrm{mg} \mathrm{ml}^{-1}\right)$, DNA was purified using a QIAquick PCR purification kit (Qiagen). ChIP primers sequences are listed here: AURKAChip-for: 5' -CGAATCCTGCCCAATCTACC3'; AURKAChip-rev: 5'-GATGGCGAGAAAAGCAAGAG-3'; ncRNAa7Chipfor: 5'-GGATAACCAAAAGCGTAGGAAA-3'; ncRNAa7Chip-rev: 5'-CGGTC TCTAACAACAACAGCTC-3'; CSTF1-Chip-for: 5' -TCCATTTTTCCAGGAG AGAGC-3'; CSTF1-Chip-rev: 5'-CCAGTCGTTTCTGTGGTTTTC-3'; TAL1Chip-for: 5' -TCCTTCCCCCTTTTCCTTAC-3'; TAL1-Chip-rev: 5' -TGAACGC ACTCTCACAATCC-3'; ncRNA-a3-Cfor: 5' -ATGAGAGCCTCGGAAGTTGA3'; ncRNA-a3-Crev: 5'-CCTTGCATTCCACAGGAAGT-3'; STIL-Chip-for: 5' AATGTTACCCACCAACCTTCC-3'; STIL-Chip-rev: 5'-CTACCCTGCAAAC AGACCTCA-3'; UBE2V1-Chip-for: 5' -TGCTTTTGGGGAGAATGAAG-3'; UBE2V1-Chip-rev: 5' -TCCTCGAACAGGAGACTGGT-3'.

Protein affinity purification and RNA immunoprecipitation. The affinitypurified Mediator complex and the GFP protein control were generated from the Flag-tagged MED12 or GFP HEK293T stable cell lines.

RNA probes, internally labelled with $\left[\alpha_{-}{ }^{32} \mathrm{P}\right] \mathrm{CTP}$, were transcribed using T7 or Sp6 RNA polymerase (Riboprobe System, Promega).

RNA immunoprecipitations were performed using Dynabeads Protein A (Invitrogen) according to manufacturer's recommendations. $2 \mu \mathrm{g}$ antibody was incubated with each sample, and the purified protein complex or the control 
protein together with labelled RNA probes were incubated with the beads at $4{ }^{\circ} \mathrm{C}$. The immunoprecipitated RNAs were extracted by TRIzol (Invitrogen) and resolved on the 6\% Novex TBE-Urea gels (Invitrogen).

For ultraviolet crosslinking experiments, the method is modified from that of ref. 28. Transient transfected HEK293T cells were UV-crosslinked at $254 \mathrm{~nm}$ $\left(200 \mathrm{~mJ} \mathrm{~cm}^{-2}\right)$ in $10 \mathrm{ml}$ ice-cold PBS and collected by scraping. Cells were incubated in lysis solution $(0.1 \%$ SDS, $0.5 \%$ NP40, $0.5 \%$ sodium deoxycholate, $400 \mathrm{U} \mathrm{ml}^{-1}$ RNase Inhibitor (Roche)) and protease inhibitor at $4{ }^{\circ} \mathrm{C}$ for $25 \mathrm{~min}$ with rotation, followed by DNase treatment ( $30 \mathrm{U}$ of DNase, $15 \mathrm{~min}$ at $37^{\circ} \mathrm{C}$ ).

In vitro transcription of ncRNA-a. In vitro transcription followed the protocol provided by the Riboprobe in vitro transcription systems (Promega); the designed oligonucleotides are listed here: ncRNA-a1-T7FOR: 5'-TAATACGACTCAC TATAGGGgcgcatctcctacggcctccag-3'; ncRNA-a1-sp6REV: 5'-ATTTAGGTGA CACTATAGAAccaagtgtcctgtgtaataggc-3'; ncRNA-a3-T7FOR: 5'-TAATACGA CTCACTATAGGGgaagttgagcttcaggcgcggctctt-3'; ncRNA-a3-sp6REV: $5^{\prime}$-ATTT AGGTGACACTATAGAAAaccagcctcagcggtgtggaata-3'; ncRNA-a7-T7FOR: 5' TAATACGACTCACTATAGGGctgtggcagagacggagaa-3'; ncRNA-a7-SP6REV: 5'-ATTTAGGTGACACTATAGAAacagccttagactgtgaaattttattg-3'; HOTAIRT7FOR: 5 '-TAATACGACTCACTATAGGGAGGCCCCAAAGAGTCTGATG TT-3'; HOTAIR-SP6REV: 5'-ATTTAGGTGACACTATAGAACTCAGGTT TTTCCAGCGTTCTC-3'. Uppercase letters inciate either the T7 or SP6 polymerase binding site, whereas lowercase letters indicate specific sequences for each ncRNA.

Kinase assays. Kinase reactions were carried out with $10-50 \mathrm{ng}$ of purified Mediator protein complex and $500 \mathrm{ng}$ human histone H3.1 recombinant (New England Biolabs) substrates in kinase buffer $(25 \mathrm{mM}$ Tris pH 8.0, $100 \mathrm{mM} \mathrm{KCl}$, $100 \mu \mathrm{M}$ ATP, $10 \mathrm{mM} \mathrm{MgCl}_{2}, 2 \mathrm{mM}$ DTT $)$ with the addition of $2.5 \mu \mathrm{Ci}\left[\gamma_{-}{ }^{32} \mathrm{P}\right] \mathrm{ATP}$ at $24^{\circ} \mathrm{C}$. Samples were resolved on a $4-12 \%$ SDS-polyacrylamide gel (Invitrogen) followed by transfer to PVDF membrane (Millipore) and autoradiography.

Chromosome conformation capture (3C). 3C assays followed the protocol from ref. 29, with minor modifications. A549 or MCF7 cells were filtered through a $70 \mu \mathrm{m}$ filter to obtain a single-cell preparation. $1 \times 10^{7}$ cells were then fixed in $1 \%$ formaldehyde for $30 \mathrm{~min}$ at room temperature, followed by $15 \mathrm{~min}$ crosslinking. The reaction was quenched with $0.25 \mathrm{M}$ glycine and cells were collected by centrifugation at $240 \mathrm{~g}$ at $4{ }^{\circ} \mathrm{C}$. The pellet was re-suspended in $0.5 \mathrm{ml}$ cold lysis buffer (10 mM Tris-HCl, pH 7.5; $10 \mathrm{mM} \mathrm{NaCl} ; 5 \mathrm{mM} \mathrm{MgCl}$; $0.1 \mathrm{mM}$ EGTA) with freshly added protease inhibitors (Roche) and was lysed on ice for $15 \mathrm{~min}$. Cells were Dounce homogenized on ice with pestle B using 15-20 strokes. The nuclei were collected by centrifugation at $500 \mathrm{~g}$ for $10 \mathrm{~min}$ at $4{ }^{\circ} \mathrm{C}$ and were re-suspended in $0.5 \mathrm{ml}$ of $1.2 \times$ restriction enzyme buffer (NEB), including $0.3 \%$ SDS and were incubated for $1 \mathrm{~h}$ at $37^{\circ} \mathrm{C}$ while shaking at 1,200 r.p.m. Two per cent (final concentration) Triton $\mathrm{X}-100$ was added to the nuclei and then the samples were incubated for $1 \mathrm{~h}$ at $37^{\circ} \mathrm{C}$ while shaking. $400 \mathrm{U}$ restriction enzyme was added to the nuclei and the samples were incubated at $37^{\circ} \mathrm{C}$ overnight while shaking. $10 \mu \mathrm{l}$ of the samples were collected before and after the enzyme reaction to evaluate digestion efficiency. The reaction was stopped by addition of 1.6\% SDS (final concentration) and incubation at $65^{\circ} \mathrm{C}$ for 30 min while shaking at 1,200 r.p.m. The sample was then diluted 10 -fold with $1.15 \times$ ligation buffer $(\mathrm{NEB})$ with added $1 \%$ Triton X-100 and was incubated for $1 \mathrm{~h}$ at $37^{\circ} \mathrm{C}$ while shaking at 900 r.p.m. 100 U T4 DNA ligase (NEB) were added to the sample and the reaction was carried at $16^{\circ} \mathrm{C}$ for $4 \mathrm{~h}$ followed by $30 \mathrm{~min}$ at room temperature. $300 \mu \mathrm{g}$ of Proteinase $\mathrm{K}$ were added to the sample and the reaction was carried at $65^{\circ} \mathrm{C}$ overnight for de-crosslink. RNA was removed by adding $300 \mu \mathrm{g}$ of RNase and incubating the sample for $1 \mathrm{~h}$ at $37^{\circ} \mathrm{C}$. DNA was purified by twice phenol-chloroform extraction and ethanol precipitation. Purified DNA was then analysed by conventional or quantitative PCR. As a control for ligation products the BAC clones were digested with $10 \mathrm{U}$ of restriction enzyme overnight and then incubated with $10 \mathrm{U}$ T4 DNAligase at $16{ }^{\circ} \mathrm{C}$ overnight. The DNA was extracted by phenol-chloroform and precipitated with ethanol. Purified DNA was then analysed by conventional or quantitative PCR. For real-time PCR, the $\Delta$ Ct method was applied for analysing data, using the $\mathrm{BAC}$ clone $\mathrm{Ct}$ values as control. $\mathrm{Ct}$ values were normalized for each primer pair by setting the $\mathrm{Ct}$ value of $100 \mathrm{ng}$ of $\mathrm{BAC}$-clone control random ligation matrix DNA at a value of 1 . Primer sequences for PCR are listed here: BackgControl-AURKA-for: 5'-CGTATGAGCTGGCAATAGCA-3'; Backg-ControlAURKA-rev: 5'-GGAGGCATGAGAGCTTGTTC-3'; Backg-Control-A7-for: 5'-ACTGGCTGAGGTTTGTGGAG-3'; Backg-Control-A7-rev: 5'-GGCCGA TTTGAGAGAGTGAG-3'; Backg-Control-SNAI1-for: 5'-CAAGAGGGGAA GGAGAGGAG-3'; Backg-Control- SNAI1-rev: 5'-TCCTAAGTCCCCAGTC TCCA-3'; ncRNA-a7 anchor primer: 5'-CTAGGTCAGGCCCAAAGAGA-3'; ncRNA-a7 anchor control: 5'-ACAGAATAACCCATCCCTTTTCC-3'; A7anchorDigTest-for: 5' -GCTTAACCCAGTGCAAGCTC-3'; A7-anchorDigTestrev: 5'-AGTTTGGAGGCTGGGAATCT-3'; AURKA-N5DigTest-for: $5^{\prime}$-CCT GATTCTCACCCTCTTGG-3'; AURKA-N5DigTest-rev: 5'-GAGGCCCCAT TCCTTAACAT-3'; SNAI1-N2DigTest-for: 5'-GGGAAAATGAGAGCAAAC TCC-3'; SNAI1-N2DigTest-rev: 5'-AAGGCCAATGTGACAGAGATG-3'; A3DigEcoRI-for: 5' -CACTTACAGGAACAGAGGACTCA-3'; A3-DigEcoRI-rev: 5'-CAACTTCTAGATCAGCGCCTTC-3'; 3C AURKA-BglII-A: 5'-GGGACAT AAATGGGAGACTGG-3'; 3C AURKA-BglII-B: 5' -TGGGAGGAACTCTTC ACCTCT-3'; 3C AURKA-BglII-C: 5' -TGGCTCTTTGGTCTTGTCTCT-3'; 3C AURKA-BglII-D: 5'-GTTTGGGCCGTTTCTTATTG-3'; 3C AURKA-BgIII-E: 5'-CAGACCTGATTCTCACCCTCTTG-3'; 3C AURKA-BglII-F: 5'-CACCAA CTTTTCTTCTCCACCAA-3'; 3C AURKA-BglII-G: 5'-CCACAGGCACTACT GTTACCAA-3'; 3C BglII.E-R1: 5'-CCAGGGAGGACACAGCATAA-3'; 3C BglII.E-R2: 5'-TGGTGTCTGGGTTGAAGTAACA-3'; 3C BglII.E-R3: 5'-TC GGCACCTGGTAAACTAGC-3'; ncRNA-a3 anchor primer: 5'-CAACTTCTA GATCAGCGCCTTC-3'; ncRNA-a3 anchor control: $5^{\prime}$-GTCTCTTTCTTTGAT TGGAGTGG-3'; TAL1-1: 5' -GGGAATGGCAGAAGACAGAA-3'; TAL1-2: 5' GTGTCGGGTTTGTTTTTACCTC-3'; TAL1-3: 5'-CTCCATTTTTGCCTCTG TCTCT-3'; TAL1-4: 5'-ATCTTTTCCGTGTAGCTCTTCCT-3'; TAL1-5: 5' TGGGTTTGATTGCTCAGTACC-3'; TAL1-6: $5^{\prime}$-TCAATTTTGCCTGCCAT ACA-3'; TAL1-7: 5'-TTAAAAGGGCAGGGAGTTAAAAG-3'; TAL1-8: 5' CAGATAGCCAGGAAGCAAGG-3'; SNAI1-1: 5'-GCTTTGGTCCTGAAC TCCTG-3'; SNAI1-2: 5' -AACAAACTCACATTAATAAAATATCCAGA-3'; SNAI1-3: 5' -CTACAGTCGTGTGCCACCAA-3'; SNAI1-4: 5' -ATTGGCAAGA AGGGGAAAAT-3'; SNAI1-5: 5' -TCCAACATCCCAGACCTTTC-3'; SNAI1-6: 5'-GACCATGAGAAAGCCAGAGG-3'; SNAI1-7: 5' -AAGCTCCAGTGGCAT GTGAT-3'; SNAI1-8: 5'-TGGAGTCCTGGTGTGTTCTG-3'. BAC clone ID: RP-11-664B18, RP-11-1067D15, RP11-742I1 (Empire Genomics).

28. Jeon, Y. \& Lee, J. T. YY1 tethers Xist RNA to the inactive $X$ nucleation center. Cell $146,119-133(2011)$

29. Hagège, H. et al. Quantitative analysis of chromosome conformation capture assays (3C-qPCR). Nature Protocols 2, 1722-1733 (2007). 